\title{
OCCURRENCE OF VANCOMYCIN-RESISTANT STAPHYLOCOCCUS AUREUS IN THE ORAL CAVITY OF PATIENTS WITH DENTAL CARIES
}

\author{
SAJITH VELLAPPALLY ${ }^{1}$, DARSHAN DEVANG DIVAKAR ${ }^{1,2} *$, \\ ABDULAZIZ ABDULLAH Al KHERAIF ${ }^{1}$, RAVIKUMAR RAMAKRISHNAIAH ${ }^{1}$, \\ AMER AlQAHTANI ${ }^{3}$, M. H. N. DALATI ${ }^{4}$, SUKUMARAN ANIL ${ }^{5}$, \\ AFTAB AHMED KHAN ${ }^{1}$ and P. R. HARIKRISHNA VARMA ${ }^{6}$ \\ ${ }^{1}$ Dental Health Department, Dental Biomaterials Research Chair, College of Applied \\ Medical Sciences, King Saud University, Riyadh 11433, Saudi Arabia \\ ${ }^{2}$ Department of Oral Medicine and Radiology, KVG Dental College and Hospital, \\ Sullia, India \\ ${ }^{3}$ Security Force Hospital, Riyadh, Saudi Arabia \\ ${ }^{4}$ Springs Dental Care, Horsforth, Leeds, UK \\ ${ }^{5}$ Department of Preventive Dental Sciences, College of Dentistry, Prince Sattam Bin \\ Abdulaziz University, Post Box: 153, Al-Kharj 11942, Saudi Arabia \\ ${ }^{6}$ Visiting Professor, College of Applied Medical Sciences, King Saud University, Riyadh, \\ Saudi Arabia
}

(Received: 9 December 2016; accepted: 10 March 2017)

Oral streptococci are the major group of microbes isolated from oral microflora. They represent frequent pathogens of infective endocarditis (IE), and it is assumed that in most of the cases oral streptococci are acquired via mucosa layer of oral cavity. Staphylococcus aureus is also frequently isolated from IE as it accounts for $20 \%-30 \%$ of all cases. Vancomycin has been the most reliable therapeutic agent against infections caused by methicillin-resistant $S$. aureus (MRSA). The main objective of this study was to examine the occurrence of $S$. aureus species in dental caries specimens. Antimicrobial susceptibility testing of $S$. aureus to four antibiotics namely vancomycin, linezolid, teicoplanin, and daptomycin was performed. Detection of vancomycin resistance was conducted using polymerase chain reaction. Among the tested 150 strains, 98 were MRSA and of that 54 were vancomycin sensitive and 27 were resistant. All 98 MRSA strains were positive for mecA and 36 yielded $p v l$, whereas 13 carried vanA and only 2 were positive for vanB. Majority of the isolates showed sensitivity toward daptomycin and linezolid. Strains of $S$. aureus exhibiting decreased susceptibility to different antibiotics like vancomycin, daptomycin, and linezolid severely compromise the therapeutic alternatives and require a considerable

*Corresponding author; E-mail: darshanddivakar@gmail.com 
amount of time, public awareness, and integrative health-care strategies to prevent the emergence of resistance to these compounds.

Keywords: antibiotic resistance, multidrug-resistant bacteria, S. aureus, vanA, dental caries, S. aureus, antibiotics, vancomycin resistant

\section{Introduction}

Multidrug-resistant strains are arising worldwide. They have become a menace, which requires more attention and a driving force to eradicate them. Methicillin-resistant Staphylococcus aureus (MRSA) has been of increased concern and a major pathogen of hospital-acquired infections [1,2]. Vancomycin was considered to be the best alternative for the treatment of multidrug-resistant MRSA. However, there have been increasing reports of vancomycin-resistant $S$. aureus (VRSA) strains exhibiting two different resistance mechanisms [3, 4]. Over the past two decades, vancomycin has been the drug of choice for MRSA, but recent studies have indicated the therapeutic failure of vancomycin against MRSA isolates. It has aroused global concern since only a limited number of effective therapeutics are currently available against MRSA [5, 6]. There have been reports of MRSA encoding both mecA and mecC confer resistance to $\beta$-lactams and poses diagnostic problems [7]. In various studies, it has been noted that MRSA strains were also resistant to one or more non- $\beta$-lactam antibiotics and some of them were multidrug-resistant [8]. Oral streptococci are the major group of microbes isolated from oral microflora and they are frequently isolated pathogens from infective endocarditis (IE). One of the clinical importance of dental caries is the inimitable site for these bacteria to enter bloodstream and increases chances to infect heart. Therefore, dental procedures and dental hygiene have been given much more attention to the predisposing conditions and to prevent streptococcal IE [9].

Besides streptococci, reports suggest a high incidence of Staphylococcus species in IE. Among them, $S$. aureus is the most frequently isolated bacterium, which accounts for $20 \%-30 \%$ of all IE cases $[10,11]$. Though influencing conditions and route of infections related to staphylococcal IE are not indicated. However, in most of cases, it is assumed that it is acquired via a mucosal surface or percutaneous route, particularly in cases associated with nosocomial infection [12].

Staphylococci are main inhabitant of skin, as well as glands of skin. These are also found to be briefly resident in the oral cavity and it is speculated that IE cases caused by them are originated in the oral cavity [13].

In this study, we examined the occurrence of $S$. aureus in dental caries specimens. In addition, in view of the abovementioned circumstances, sensitivity 
patterns of $S$. aureus to four different antibiotics - vancomycin, linezolid, teicoplanin, and daptomycin were analyzed. The prevalence of vancomycin resistance was detected using polymerase chain reaction (PCR).

\section{Materials and Methods}

\section{Bacterial isolates}

A total of $150 \mathrm{~S}$. aureus isolates were obtained from dental caries clinical samples from the patients who were visited the facilities of dental hospitals clinic. The morphology and characteristics of $S$. aureus strains were studied using Gram staining, hemolysis on blood agar, DNase, catalase, coagulase tests, and fermentation of mannitol. The study was designed and permitted by the Institutional Ethics Committee and a written informed consent was obtained from every individual before the sample collection.

\section{Phenotypic methods for detection of MRSA}

All the isolates were subjected to disk diffusion susceptibility testing to oxacillin $(1 \mu \mathrm{g})$ and cefoxitin $(30 \mu \mathrm{g})$ disks for detection of methicillin resistance. Growth on oxacillin screen agar containing $6 \mu \mathrm{g} / \mathrm{ml}$ of oxacillin was also checked on all the isolates. MRSA (ATCC 43300) was used as positive control and S. aureus (ATCC 25923) as negative control. MRSA strains were further tested.

\section{Determination of minimum inhibitory concentration (MIC)}

MIC was determined based on Clinical and Laboratory Standards Institute guidelines. Briefly, gradient plates of Mueller-Hinton agar (Hi Media Laboratories, Mumbai, India) were prepared with vancomycin, daptomycin, teicoplanin, and linezolid (0.5-256 $\mu \mathrm{g} / \mathrm{ml}$, Sigma-Aldrich). An amount of $0.5 \mathrm{McFarland}$ equivalent inoculum prepared using 18- to 24-h-old culture was spotted on to gradient plates and the plates were incubated overnight at $35^{\circ} \mathrm{C}$ for $24 \mathrm{~h}$ to achieve visible growth.

\section{PCR amplification for all vancomycin-resistant genes}

A single target PCR was carried out to detect the presence of genes encoding vancomycin resistance. Of the many genotypes of vancomycin resistance described in enterococci, attempt was made to identify the commonest ones, PCR 
amplification of the vanA gene was performed using GeneAmp thermal cycler (Applied Biosystems, Wilmington, NC, USA).

\section{PCR for MRSA detection}

$m e c A$ gene encoding methicillin resistance and $p v l$ gene presented in community-associated MRSA strains were tested in all the 98 MRSA isolates using the primers given in Table I.

The amplification conditions maintained were initial denaturation at $98^{\circ} \mathrm{C}$ for $10 \mathrm{~s}$, annealing for $1 \mathrm{~min}$ at different temperature respective to target $\mathrm{PCR}$, polymerization was performed at $72{ }^{\circ} \mathrm{C}$ for $1 \mathrm{~min}$, and finally extension at $72{ }^{\circ} \mathrm{C}$ for $5 \mathrm{~min}$.

\section{Results and Discussion}

During the study period, a total number of 150 isolates were collected from dental caries clinical samples. Altogether 98 were found to be MRSA by disk diffusion test. Among the 98 patients, 59 of them had prolonged hospital stay of longer than 10-day duration, 42 patients had postoperative dental infections, and 29 patients were admitted due to dental infections for more than 2 days. In all, 71 patients were males and 27 patients were females.

The results of MIC for 98 isolates showed vancomycin was $<2 \mu \mathrm{g} / \mathrm{ml}$ in case of 54 isolates and 27 isolates showed intermediate resistance in the range of 4-8 $\mu \mathrm{g} / \mathrm{ml}$, whereas 15 isolates showed MIC in the range of $16-128 \mu \mathrm{g} / \mathrm{ml}$ indicating that these were VRSA. Detailed results are shown in Table II.

By PCR amplification, mecA gene was present in all the 98 isolates of MRSA and $p v l$ gene was present in 36 isolates. vanA gene was present in 13 isolates, and only 2 were $\operatorname{van} B$-positive (Figure 1).

Table I. List of primers used in this study

\begin{tabular}{lllc}
\hline Gene & \multicolumn{1}{c}{ Forward } & \multicolumn{1}{c}{ Reverse } & Reference \\
\hline vanA & AATAGCGCGGACGAATTGGAC & AACGCGGCACTGTTTCCCAA & {$[14]$} \\
vanB & CTTAACGCTGCGATAGAAGC & CTGATGGATGCGGAAGATAC & {$[14]$} \\
vanD & TTTGTAAAGCCTGCCCGTTC & CCAAGTAYCCGGTAAATCTTC & {$[14]$} \\
vanE & AAATAATGCTCCATCAATTTGCTGA & ATAGTCGAAAAAGCCATCCACAAG & {$[14]$} \\
vanG & TTGGAGGCAATTCAACAGAGT & TCGCAGCCAACAACAGGTATT & {$[14]$} \\
mecA & GTAGAAATGACTGAACGTCCGATAA & CCAATTCCACATTGTTCGGTCTAA & {$[15]$} \\
pvl & ATCATTAGGTAAAATGTCTGGACATGA & GCATCAASTGTATTGGATAGCAAAAGC & {$[15]$} \\
\hline
\end{tabular}


Table II. List of positive isolates with their antibiogram and PCR results

\begin{tabular}{llll}
\hline Isolates & $\begin{array}{c}\text { Vancomycin- } \\
\text { resistant gene }\end{array}$ & \multicolumn{1}{c}{ Resistance } & \multicolumn{1}{c}{ Susceptibility } \\
\hline VRSA1 & vanB & Vancomycin & $\begin{array}{c}\text { Daptomycin, linezolid, and } \\
\text { teicoplanin }\end{array}$ \\
VRSA2 & vanA & Daptomycin, vancomycin, and teicoplanin & $\begin{array}{l}\text { Linezolid } \\
\text { VRSA3 }\end{array}$ \\
vanA & Daptomycin, vancomycin, and teicoplanin & Linezolid \\
VRSA4 & vanA & Vancomycin and teicoplanin & Linezolid and daptomycin \\
VRSA5 & vanA & Vancomycin, daptomycin, and teicoplanin & Linezolid \\
VRSA & vanB & Vancomycin & Linezolid, daptomycin, and \\
VRSA7 & vanA & Vancomycin and teicoplanin & teicoplanin \\
VRSA8 & vanA & Vancomycin and teicoplanin & Linezolid and daptomycin \\
VRSA9 & vanA & Vancomycin and teicoplanin & Linezolid and daptomycin \\
VRSA10 & vanA & Vancomycin and teicoplanin & Linezolid and daptomycin \\
VRSA11 & vanA & Vancomycin and teicoplanin & Linezolid and daptomycin \\
VRSA12 & vanA & Vancomycin, daptomycin, and teicoplanin & Linezolid \\
VRSA13 & vanA & Vancomycin, daptomycin, and teicoplanin & Linezolid \\
VRSA14 & vanA & Vancomycin, daptomycin, and teicoplanin & Linezolid \\
VRSA15 & vanA & Vancomycin, daptomycin, and teicoplanin & Linezolid \\
\hline
\end{tabular}

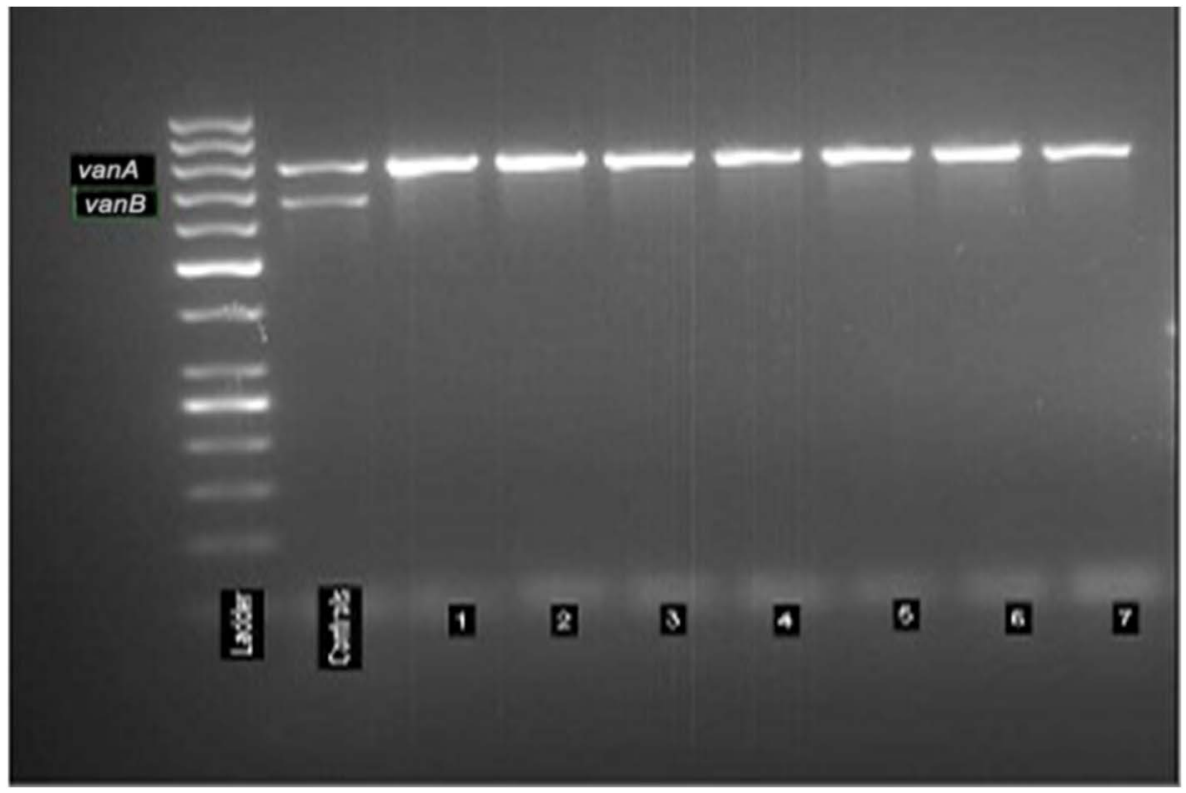

Figure 1. PCR amplification of vanA and vanB genes 
For remaining antibiotics, the range of MIC was $<1 \mathrm{~g} / \mathrm{ml}$ for daptomycin, $<8 \mathrm{~g} / \mathrm{ml}$ for teicoplanin, and $<4 \mathrm{~g} / \mathrm{ml}$ for linezolid and it was found that majority of the isolates showed sensitivity toward daptomycin and linezolid antimicrobials and only few were resistant isolates. The isolates, which were vanA-positive, have been illustrated in Table I. All these isolates were found to be highly resistant to all the antibiotics, which were tested in this study.

The rising risk of MRSA strains has proved to be a great problem globally and a positive and healthy approach has to be taken to combat this disease scourge. Vancomycin has been the most reliable therapeutic agent against infections caused by MRSA. A certain group of $S$. aureus, designated hetero-VRSA, frequently generate VRSA upon exposure to vancomycin, and are associated with infections that are potentially refractory to vancomycin therapy [16]. Although vanA-positive isolates have been found earlier in enterococci, but presence of vanA gene in $S$. aureus poses a great challenge, and it is considered to be due to conjugative transfer of the gene from enterococci to $S$. aureus during hospital-acquired infections [17].

S. aureus is the most commonly isolated Staphylococcus species in IE cases [18, 19], Staphylococcus epidermidis and Staphylococcus lugdunensis are also predominant causative agents of IE $[11,19]$, and aggressive clinical courses with high mortality [20]. Both $S$. aureus and $S$. epidermidis are found in patients with dental infection (particularly abscess); however, the possible presence of $S$. aureus is especially important in dental care due to its increased resistance. Earlier literature states that $S$. aureus amplified significantly after surgical induction of hyposalivation [14].

Among collected 150 isolates, 98 (65.3\%) were MRSA and 42 (28\%) were resistant to vancomycin. All $98 \mathrm{MRSA}$ isolates were positive for mecA gene and only $13(8.6 \%)$ and $2(2.13 \%)$ harbored vanA and vanB, respectively. About 13 patients had vanA-positive strains, which indicate that recommended infection control strategies worked and prevented further spread to other patients, but still the exact mechanism of pathogenesis remains unknown. Most of the VRSA strains were also oxacillin or methicillin resistant (MRSA).

\section{Conclusions}

Strains of $S$. aureus exhibiting decreased susceptibility or resistance to vancomycin, daptomycin, and linezolid severely compromise the therapeutic alternatives for life-threatening MRSA infections. Strategies to prevent emergence of resistance to these compounds in MRSA appear to be tough: first, an adequate decrease in the bacterial load by timely surgical drainage of 
collections and second, optimization of the pharmacological parameters of the antibiotics in specific clinical situations. An additional strategy could be a combination of treatments used against the widespread evolution of MRSA strains. Vancomycin resistance is thought to be due to increased overuse of the antibiotic in cases such as in pseudomembranous colitis or it may be due to certain changes in the bacterial cell wall structure such as thickening of the cell wall or changes in the site of action of the glycopeptides [15]. Preventing the scourge of multidrug-resistant strains particularly MRSA and VRSA will require a considerable amount of time, public awareness, and an integrative health-care approach.

\section{Acknowledgements}

The authors are grateful to the deanship of Scientific Research, King Saud University for funding through Vice Deanship of Scientific Research Chairs.

\section{Conflict of Interest}

The authors certify that there is no conflict of interest with any financial organization regarding the material discussed in the article.

\section{References}

1. Deresinski, S.: Methicillin-resistant Staphylococcus aureus: An evolutionary, epidemiologic, and therapeutic odyssey. Clin Infect Dis 40, 562-573 (2005).

2. King, M. D., Humphrey, B. J., Wang, Y. F., Kourbatova, E. V., Ray, S. M., Blumberg, H. M.: Emergence of community-acquired methicillin-resistant Staphylococcus aureus USA 300 clone as the predominant cause of skin and soft-tissue infections. Ann Intern Med 144, 309-317 (2006).

3. Thati, V., Shivannavar, C. T., Gaddad, S. M.: Vancomycin resistance among methicillin resistant Staphylococcus aureus isolates from intensive care units of tertiary care hospitals in Hyderabad. Indian J Med Res 134, 704 (2011).

4. Azimian, A., Havaei, S. A., Fazeli, H., Naderi, M., Ghazvini, K., Samiee, S. M., Soleimani, M., Peerayeh, S. N.: Genetic characterization of a vancomycin-resistant Staphylococcus aureus isolate from the respiratory tract of a patient in a university hospital in northeastern Iran. J Clin Microbiol 50, 3581-3585 (2012).

5. Howden, B. P., Davies, J. K., Johnson, P. D., Stinear, T. P., Grayson, M. L.: Reduced vancomycin susceptibility in Staphylococcus aureus, including vancomycin-intermediate and heterogeneous vancomycin-intermediate strains: Resistance mechanisms, laboratory detection, and clinical implications. Clin Microbiol Rev 23, 99-139 (2010). 
6. Lodise, T. P., Graves, J., Evans, A., Graffunder, E., Helmecke, M., Lomaestro, B. M., Stellrecht, K.: Relationship between vancomycin MIC and failure among patients with methicillin-resistant Staphylococcus aureus bacteremia treated with vancomycin. Antimicrob Agents Chemother 52, 3315-3320 (2008).

7. Paterson, G. K., Harrison, E. M., Holmes, M. A.: The emergence of mecC methicillinresistant Staphylococcus aureus. Trends Microbiol 22, 42-47 (2014).

8. Lepsanovic, Z., Jeremic, L. P., Lazic, S., Cirkovic, I.: High prevalence and resistance patterns of community-associated methicillin-resistant Staphylococcus aureus in the Pomoravlje Region, Serbia. Acta Microbiol Immunol Hung 63, 83-92 (2016).

9. Ohara-Nemoto, Y., Haraga, H., Kimura, S., Nemoto, T. K.: Occurrence of staphylococci in the oral cavities of healthy adults and nasal-oral trafficking of the bacteria. J Med Microbiol 57, 95-99 (2008).

10. Cecchi, E., Forno, D., Imazio, M., Migliardi, A., Gnavi, R., Dal Conte, I., Trinchero, R.: New trends in the epidemiological and clinical features of infective endocarditis: Results of a multicenter prospective study. Ital Heart J 5, 249-256 (2004).

11. Nakatani, S., Mitsutake, K., Hozumi, T., Yoshikawa, J., Akiyama, M., Yoshida, K., Ishizuka, N., Nakamura, K., Taniguchi, Y., Yoshioka, K., Kawazoe, K., Akaishi, M., Niwa, K., Nakazawa, M., Kitamura, S., Miyatake, K.; Committee on Guideline for Prevention and Management of Infective Endocarditis, Japanese Circulation Society: Current characteristics of infective endocarditis in Japan: An analysis of 848 cases in 2000 and 2001. Circ J 67, 901-905 (2003).

12. Di Filippo, S., Delahaye, F., Semiond, B., Celard, M., Henaine, R., Ninet, J., Sassolas, F., Bozio, A.: Current patterns of infective endocarditis in congenital heart disease. Heart $\mathbf{9 2}$, 1490-1495 (2006).

13. Ikeda, Y., Ohara-Nemoto, Y., Kimura, S., Ishibashi, K., Kikuchi, K.: PCR-based identification of Staphylococcus epidermidis targeting gseA encoding the glutamic-acid-specific protease. Can J Microbiol 50, 493-498 (2004).

14. Yamashita, K., Ohara, M., Kojima, T., Nishimura, R., Ogawa, T., Hino, T., Okada, M., Toratani, S., Kamata, N., Sugai, M., Sugiyama, M.: Prevalence of drug-resistant opportunistic microorganisms in oral cavity after treatment for oral cancer. J Oral Sci 55, 145-155 (2013).

15. Appelbaum, P. C.: The emergence of vancomycin intermediate and vancomycin resistant Staphylococcus aureus. Clin Microbiol Infect 12, 16-23 (2006).

16. Hiramatsu, K.: Vancomycin-resistant Staphylococcus aureus: A new model of antibiotic resistance. Lancet Infect Dis 1, 147-155 (2001).

17. Moellering, R. C.: Vancomycin-resistant enterococci. Clin Infect Dis 26, 1196-1199 (1998).

18. Hoen, B., Alla, F., Selton-Suty, C., Béguinot, I., Bouvet, A., Briançon, S., Casalta, J. P., Danchin, N., Delahaye, F., Etienne, J., Le Moing, V., Leport, C., Mainardi, J. L., Ruimy, R., Vandenesch, F.: Association pour l'Etude et la Prévention de l'Endocardite Infectieuse (AEPEI) Study Group: Changing profile of infective endocarditis: Results of a 1-year survey in France. JAMA 288, 75-81 (2002).

19. Niwa, K., Nakazawa, M., Tateno, S., Yoshinaga, M., Terai, M.: Infective endocarditis in congenital heart disease: Japanese national collaboration study. Heart 91, 795-800 (2005). 
20. Anguera, I., Del Río, A., Miró, J. M., Matínez-Lacasa, X., Marco, F., Gumá, J. R., Quaglio, G., Claramonte, X., Moreno, A., Mestres, C. A., Mauri, E., Azqueta, M., Benito, N., García-de la María, C., Almela, M., Jiménez-Expósito, M. J., Sued, O., De Lazzari, E., Gatell, J. M.: Hospital Clinic Endocarditis Study Group: Staphylococcus lugdunensis infective endocarditis: Description of 10 cases and analysis of native valve, prosthetic valve, and pacemaker lead endocarditis clinical profiles. Heart 91, e10 (2005). 